\title{
White-donkey: búsqueda de personas con vehículos aéreos no tripulados basada en visión por computadora
}

Jesús Jaime Moreno Escobar, Edgar Antonio Domínguez Ramírez, Jesús Cruz Hernández, Oswaldo Morales Matamoros, Ricardo Tejeida Padilla

\author{
Instituto Politécnico Nacional, \\ México \\ jemoreno@esimez.mx
}

\begin{abstract}
Resumen. La búsqueda de una persona extraviada no es una tarea sencilla de llevar a cabo, por lo que a lo largo de los años se han desarrollado metodos de busqueda, el problema es que los métodos actualmente existentes cuentan con ciertas limitantes y estas limitantes se ven reflejadas en el tiempo de localización. En la búsqueda de personas el tiempo de localización es un factor muy importante que los rescatistas no se pueden dar el lujo de desperdiciar ya que la persona extraviada está expuesta a grandes peligros. Haciendo uso de la visión por computadora se espera realizar la detección y búsqueda de alguna persona extraviada. Así, se diseñaron e implementaron algoritmos para que un Vehículo aéreo no tripulado o UAV (Unmanned Aerial Vehicle) lleve a cabo esta tarea, además gracias a la velocidad de este se espera aminorar el tiempo de localización. Con el uso de un UAV no se pretende reemplazar al ser humano en la difícil tarea de buscar y rescatar personas sino que se pretende que sirva como una herramienta de apoyo en la realización de esta difícil tarea.
\end{abstract}

Palabras clave: Visión por computadora, vehículos aéreos no-tripulados, sistemas de búsqueda y rescate, sistema visual humano, cuadricópteros.

\section{White Donkey: People Search with Unmanned Aerial Vehicles based on Computer Vision}

\begin{abstract}
Searching for a missing person is not an easy task to accomplish,so over the years search methods have been developed, the problem is that the methods currently available have certain limitations and these limitations are reflected in time location. Time location in a person search is a very important factor that rescuers cannot afford to waste because the missing person is exposed to great dangers. Using computer vision is expected to search for any missing person designing and implementing algorithms in order to an Unmanned Aerial Vehicle perform this task, also thanks to the speed of this is expected to reduce the time location.
\end{abstract}


By using of a Unmanned Aerial Vehicle is not intended to replace the human being in the difficult task of searching and rescuing people but rather is intended to serve as a support tool in performing this difficult task.

Keywords: Computer vision, unmanned aerial vehicles, search and rescue systems, human visual system, quadricopters.

\section{Introducción}

El uso de drones en situaciones de emergencia cada vez es más frecuente, debido a que los drones permiten el acceso de manera efectiva, rápida a la hora de acceder a todo tipo de terrenos desconocida, además de desplazarse fácilmente por terrenos irregulares gracias a su gran estabilidad. Además es posible equipar a los drones con cámaras de alta definición por lo que el equipo de rescate tiene la facilidad de dirigir los drones haciendo uso de computadores portátiles y equipos de control [3]. El equipo de rescate hace uso de drones para tareas de búsqueda de personas desaparecidas. A continuación se mencionan algunos ejemplos.

España destaca dentro de este ramo, a continuación se citan tres ejemplos:

1. Ya se han utilizado drones de forma efectiva en Zaragoza en la búsqueda de personas desaparecidas por parte de una empresa privada que ha colaborado con la policía aragonesa,

2. La dotación de bomberos de valencia ya está utilizando drones en labores de búsqueda de personas desaparecidas con éxito,

3. El departamento de ingeniería de sistemas y automática de la universidad de Málaga está realizando un proyecto de investigación en el cual une los equipos de rescate canino con drones, estos drones hacen uso de cámaras térmicas Figura 1(a).

Por otro lado en enero de 2014, Intel y Ascending Technologies presentaron prototipos de drones multicópteros que son capaces de desplazarse por una pista de obstáculos y evitar automáticamente a quien encuentre en su camino. Los prototipos utilizan el módulo de cámara RealSense de Intel, cuyo peso es de solo 8 g y su grosor menor a $4 \mathrm{~mm}$.

Mientras tanto en México, en el estado de Morelos la cruz roja mexicana firmo un convenio con la empresa SkyBotica para el uso de un grupo de drones Figura 1(b). Estos se emplearan en la tarea de búsqueda y rescate de personas extraviadas

\section{Marco teórico}

\subsection{Visión por computadora: algoritmo Viola \& Jones}

Este algoritmo tiene un bajo coste en hardware y consta de dos partes principales: Clasificador en cascada el cual garantiza una discriminación rápida 
White-donkey: búsqueda de personas con vehículos aéreos no tripulados basada en visión ...
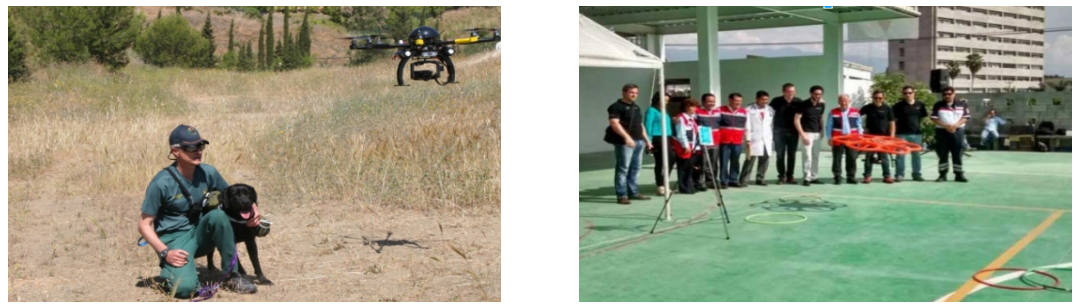

Fig. 1. (a) Drones en Málaga y (b) Cruz roja mexicana y SkyBotica.

y un entrenador de clasificadores basado en Adaboost [2]. Viola Jones tiene una probabilidad de verdaderos positivos del $99.9 \%$ y una probabilidad de falso positivos del $3.33 \%$ y a diferencia de otros algoritmos utilizados en métodos de caracteres invariantes, este sólo procesa la información presente en una imagen en escala de grises. No utiliza directamente la imagen sino que utiliza una representación de esta llamada imagen integral.

Para determinar si en una imagen se encuentra una cara o no, el algoritmo divide la imagen integral en subregiones de tamaños diferentes y utiliza una serie de clasificadores (clasificadores en cascada), cada una con un conjunto de características visuales. En cada clasificador se determina si la subregión es una cara o no. El uso de este algoritmo supone un ahorro de tiempo considerable ya que no serán procesadas subregiones de la imagen que no se sepa con certeza que contienen una cara y sólo se invertirá tiempo en aquellas subregiones que posiblemente sí contengan una cara, Figura 2.

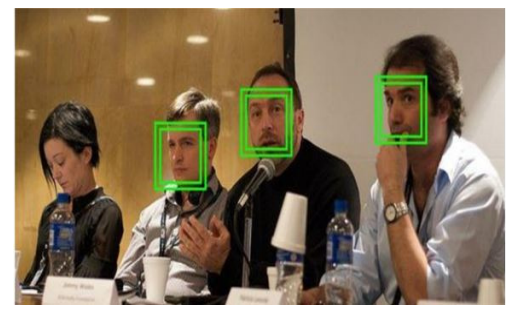

Fig. 2. Detección facial por medio del Algoritmo Viola \& Jones.

\subsection{Principio de operación del tetramotor}

Un tetramotor es un sistema multivariable que cuenta con seis grados de libertad (x, y, z,pitch, roll y yaw) y subactuado ya que consta de menos actuadores que grados de libertad. Los rotores del tetramotor producen las fuerzas y momentos principales que actúan sobre este, Figura 3 [1]. El balance del torque total del sistema se logra debido a que dos pares de motores rotan en direcciones 
opuestas. Para el estudio del sistema se utilizan dos sistemas de referencia el sistema T fijo a tierra y el sistema B fijo al vehículo [4].
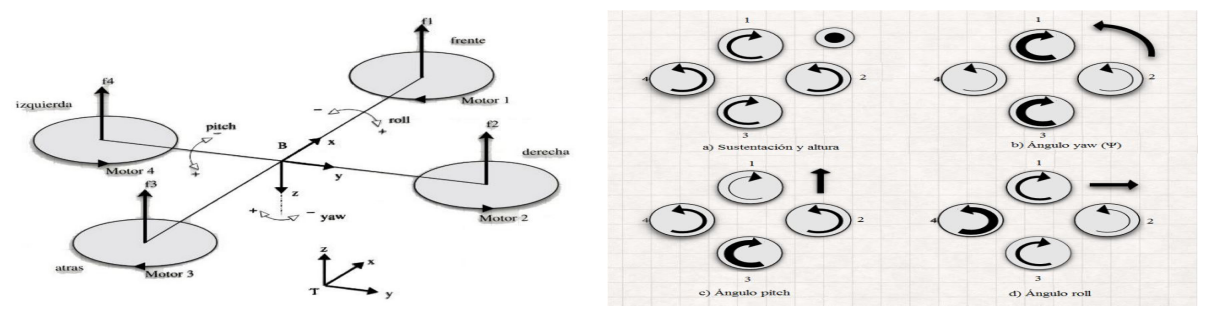

Fig. 3. Operación del tetramotor: (a) Sistemas de referencia y (b) Su dinámica.

La dinámica de un tetramotor se puede resumir en los siguientes 4 puntos:

1. Sustentación y altura: Los cuatro motores giran con la misma potencia y en direcciones opuestas.

2. Diferencia en torque para manipular yaw $(\psi)$ : Para girar hacia la derecha sobre su propio eje, los motores 1 y 3 aumentan su potencia girando ambos hacia el mismo sentido. Para girar hacia la izquierda sobre su propio eje, los motores 2 y 4 aumentan su potencia girando ambos hacia el mismo sentido.

3. Diferencia en torque para manipular pitch $(\theta)$ : Para avanzar hacia adelante, los motores 2 y 4 aumentan de potencia, de igual forma el motor 3 aumenta su potencia pero a un grado mayor que los motores 2 y 4 . Para avanzar hacia atrás en lugar de que el motor 3 aumente el que aumenta es el motor 1.

4. Diferencia en torque para manipular roll $(\varphi)$ : Para avanzar hacia la derecha, los motores 1 y 3 aumentan de potencia, de igual forma el motor 4 aumenta su potencia pero a un grado mayor que los motores 1 y 3 . Para avanzar hacia la izquierda en lugar de que el motor 4 aumente el que aumenta es el motor 2 .

Los motores utilizados en este proyecto son de tipo brushless. Un motor eléctrico brushless (sin escobillas) se define como un motor eléctrico que no emplea escobillas para realizar el cambio de polaridad en el rotor. Los motores brushless están compuestos por una parte móvil, el rotor, que es donde se encuentran los imanes permanentes y una parte fija denominada estator o carcasa sobre la cual van dispuestos los bobinados de hilo conductor. Para hacer un funcionar un motor brushless es necesario el uso del electronic speed control (ESC). Un electronic speed control (control electrónico de velocidad o ESC) es un circuito electrónico que tiene como función variar la velocidad de un motor eléctrico, su dirección y posiblemente también para actuar como un freno dinámico. Los ESC son comúnmente utilizados en los motores accionados eléctricamente por radio control, con la variedad más utilizada para motores brushless, proporcionando una fuente generada electrónicamente de tres fases de bajo voltaje. Un ESC 
White-donkey: búsqueda de personas con vehículos aéreos no tripulados basada en visión ...

puede ser una unidad independiente que se conecta en el canal del control de aceleración en el receptor o puede ya estar incorporado en el propio receptor, como es el caso en la mayoría de los vehículos de juguete de calidad de R / C.

La potencia de los tetramotores será controlada por un transceptor, cuya definición es la siguiente: Un transceptor es un dispositivo que cuenta con un transmisor y un receptor que comparten partes de su propio circuito. Cuando el transmisor y el receptor no tienen en común partes del circuito electrónico se conoce como transmisor-receptor. Dado que determinados elementos del circuito se utilizan tanto para la transmisión como para la recepción, la comunicación que provee un transceptor solo puede ser semidúplex, lo que significa que pueden enviarse señales en ambos sentidos, pero no simultáneamente. El Transceptor RF1100 es un módulo de RF a una frecuencia de $433 \mathrm{MHz}$. El módulo transmite de forma inalámbrica a velocidades de transmisión de 4800, 9600 y 19200 bit/s, tiene 256 canales individuales que pueden ser seleccionados. Este módulo soporta la transmisión punto a multipunto.

\section{Metodología}

\subsection{Descripción general del sistema}

Para el estudio y planteamiento de la presente Metodología es necesario conocer los diferentes métodos existentes que realizan la detección de objetos mediante técnicas de visión por computadora. También, los principios de operación de los elementos principales que conforman al UAV para su diseño y armado.

Este sistema busca personas en el entorno haciendo uso de vehículos aéreos no tripulados y de técnicas de visón por computadora. En la actualidad ya se hace uso de drones para buscar personas pero estos drones son manipulados por los rescatistas, ya sea por medio de control remoto o con una computadora portátil. El rescatista maneja el dron para detectar personas en el entorno.

El presente sistema hace uso de algoritmos de visón por computadora para que el sistema de visón del dron detecte a una persona de manera autónoma por lo que mientras que el dron lleva a cabo esta tarea, el rescatista puede realizar otras acciones y una vez que el sistema de visión del dron encuentra a una persona en el entorno, este le manda una alerta al rescatista además de que el dron se dirige hacia la persona detectada para que de esta manera el rescatista pueda obtener más detalles de la persona y del entorno.

Para darle autonomía al dron se diseñó e implemento un control digital para este con el fin de poder controlarlo a partir una computadora. Al decir poder controlarlo a través de una computadora no hace referencia a que un usuario manipula al dron, si no que a través de los datos que se obtengan a través del procesamiento de imágenes, la computadora le manda las acciones a realizar al dron, es decir, el sistema de visón con el que cuenta el dron no solo es para detectar personas, si no que también son los ojos de este.

En la Figura 4 se muestra el sistema propuesto de manera general, el cual está dividido en tres Fases principales: 


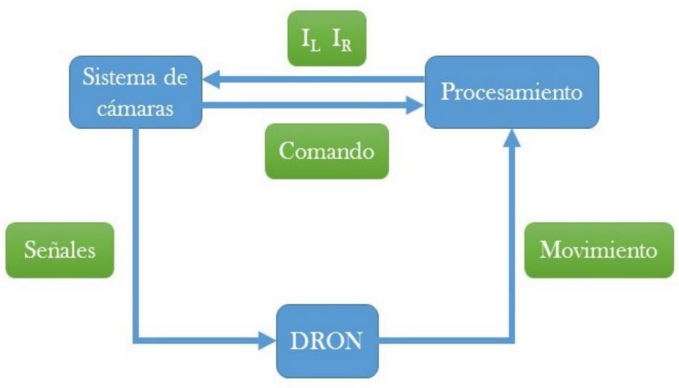

Fig. 4. Sistema General de WHITE - DONKEY.

1. Sistema de Cámaras o Fase de Captura,

2. Procesamiento Digital de Señales e Imágenes,

3. UAV o Interfaz de Cotrol.

Es importante resaltar que se realizó una Interfaz de Usuario para conjutar las tres fases, la cual se considera una fase paralela a las principales.

\subsection{Fase de captura}

Se realiza el diseño del sistema de visión estereoscópico para ello, se hace uso de la nueva tecnología de modelado e impresión 3D para la construcción de una base donde estarán sujetas las dos cámaras, con una distancia de $10 \mathrm{~cm}$ entre ellas. Ahora es necesario calibrar las cámaras que conforman al sistema de visión, es decir, obtener los parámetros intrínsecos y extrínsecos de ambas cámaras y así como los parámetros del sistema estereoscópico, Figura 5. Así, para calibrar el sistema de visión se utiliza el método de Zhang [5], este método ya está implementado en el software Matlab por lo que para utilizarlo basta con llamar a la función en Matlab con el comando cameraCalibrator, una vez realizada la calibración esta herramienta devuelve un objeto con los parámetros del sistema estereoscópico.
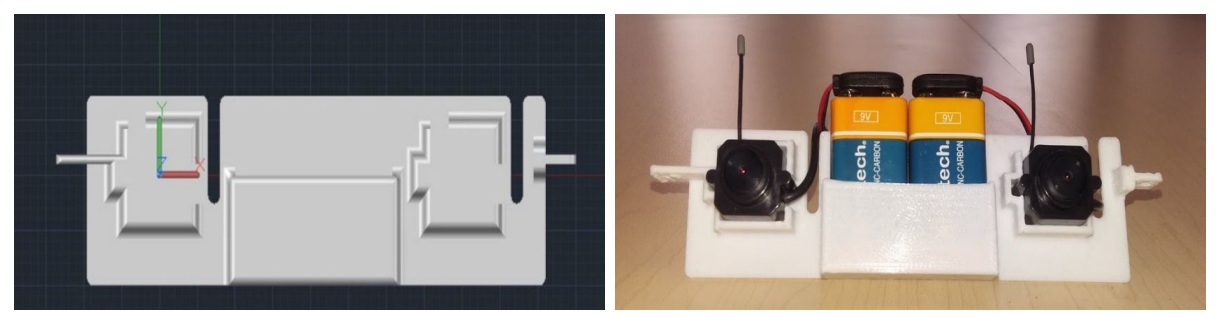

Fig. 5. Modelado en 3D de la base del sistema de visión estereoscópica. 
White-donkey: búsqueda de personas con vehículos aéreos no tripulados basada en visión ...

Ya estando listo el sistema de visión para detectar una persona en una imagen se utiliza el algoritmo Viola-Jones el cual ya viene implementado en Matlab.

A continuación se muestra el algoritmo de detección de persona y rostro:

1. Inicio.

2. Configurar la cámara 1.

3. Abrir y Configurar el puerto serial.

4. Tomar una foto del entorno y guárdarla en I.

5. Definir y configurar el detector de objetos utilizando el constructor bodyDetector, como imagen de entrada $I$ nombrarlo bboxBody.

6. Definir y configurar el detector de objetos utilizando el constructor faceDetector, como imagen de entrada $I$ y nombrarlo bboxes.

7. Si el contenido de bboxes o boxBody es mayor a cero dibujar un rectángulo en la imagen $I$ del tamaño y coordenadas bboxes también dibujar un rectángulo del tamaño y coordenadas bboxBody.

8. Si no hay contenido en bboxes mandar un carácter específico por el puerto serial.

También se plantea la metodología general para encender un LED si se detectó una persona, se lleva a cabo en el microcontrolador MSP430G2553:

1. Inicio.

2. Configurar entradas

3. Configurar salidas.

4. Configurar la comunicación serial.

5. Si el MSP430G2553 en el registro UCA0RXBUF recibe un carácter específico encender un led.

6. Si el MSP420G2553 en el registro UCA0RXBUF recibe un carácter específico apagar el led.

Así para medir la distancia hacia la persona detectada, se propone el siguiente algoritmo:

1. Inicio.

2. Cargar los parámetros de las cámaras, camStereoParams.

3. Tomar foto con la cámara derecha, guardarla en frameRigth.

4. Tomar foto con la cámara izquierda, guardarla en frameLeft.

5. Rectificación estereoscópica de frameLeft y frameRigth haciendo uso de camStereoParams, guardar las nuevas imágenes en frameLeftRect y frameRightRect.

6. Pasar frameLeftRect y frameRightRect a escala de grises y guardarlas en frameLeftGray y frameRigthGray.

7. Detectar cara en la imagen frameRightGray y en la imagen frameLeftGray.

8. Encontrar los centros de los rostros detectados.

9. Triangulación usando los centros de los rostros detectados.

10. Calcular la distancia en metros

11. Si la distancia es menor de dos metros mandar un carácter específico por el puerto serial.

12. Si la distancia es mayor de dos metros mandar un carácter específico por el puerto serial

13. Si no hay persona mandar un carácter específico por el puerto serial.

\subsection{Interfaz de control}

Debido a la complejidad de diseño, el tiempo que toma la creación desde cero de un UAV y el poder controlarlo desde una computadora, se decidió utilizar la tarjeta de control kk2.0. Esta tarjeta es utilizada en diferentes vehículos aéreos, tales como:
1. Tricopter
2. Quadcopter +
3. Quadcopter X
4. Hexcopter +
5. Hexcopter X
6. Octocopter +
7. Octocopter X
8. Aero $1 \mathrm{~S}$ Aileron
9. Aero $2 \mathrm{~S}$ Aileron
10. Flying Wing
11. Singlecopter $2 \mathrm{M} 2 \mathrm{~S}$
12. Singlecopter $1 \mathrm{M} 4 \mathrm{~S}$ 
La tarjeta de control kk2.0 tiene 6 grados de libertad que es bastante sensible. También el MPU6050 consta de un giroscopio y un acelerómetro. Esta tarjeta es controlada con el receptor hobbyking hk-t6a, el problema de este receptor radica en la frecuencia ya que es la misma en la que las cámaras transmiten, lo cual provoca demasiada interferencia haciendo imposible la detección de personas. La solución a este problema es remplazar el receptor y el control, en su lugar se realizará un circuito que entregue las mismas señales que el receptor entrega a la tarjeta de control Tabla 1, solo se utilizan 5 canales de los 6 disponibles:

Tabla 1. Valores medidos de cada canal que entrega el receptor.

\begin{tabular}{|c|c|c|c|}
\hline Canal & Función & TH & TL \\
\hline 1 & Aileron & $1.54 \mathrm{mS}$ & $16.79 \mathrm{mS}$ \\
\hline 2 & Elevator & $1.54 \mathrm{mS}$ & $16.79 \mathrm{mS}$ \\
\hline 3 & Throttle & $1.02 \mathrm{mS}$ & $17.33 \mathrm{mS}$ \\
\hline 4 & Ruder & $1.54 \mathrm{mS}$ & $16.79 \mathrm{mS}$ \\
\hline 5 & Aux & $2 \mathrm{mS}$ & $16 \mathrm{mS}$ \\
\hline
\end{tabular}

Así, se propone el siguiente algoritmo para controlar los movimientos el UAV a distancia:

- Inicio.

- Configuración de registros para comunicación serial.

- Configuración de los timers.

- Generar 3 PWM's utilizando el timer.

- Con cada carácter recibido en el registro UAC0RXBUF de la comunicaciones serial, el ciclo útil cambiaran según el carácter.

\subsection{Interfaz de usuario}

Para que el uso del software de búsqueda de personas sea amigable al usuario, se diseña una interfaz gráfica de usuario (GUI, Figura 6) la cual se diseñada con la herramienta GUI que matlab proporciona. En esta aplicación el usuario introduce el puerto de comunicación con el cual se enlaza al transmisor con la computadora. La aplicación cuenta con el panel de ARMED Y SEARCHING. El panel de ARMED nos permite establecer la comunicación con el UAV y el panel SEARCHING nos permite comenzar o detener la búsqueda. Finalmente contamos con una ventana en la cual se muestra a la persona detectada, un indicador gráfico y uno textual. Cuando se detecta a una persona el indicador gráfico está en color verde y el indicador textual indica DETECTED PERSON, cuando no se detecta nada el indicador gráfico está en color rojo y el indicador textual indica UNDETECTED PERSON. 
White-donkey: búsqueda de personas con vehículos aéreos no tripulados basada en visión ...

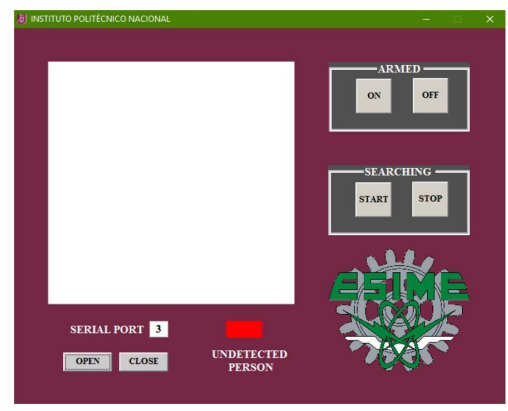

Fig. 6. Interfaz de usuario.
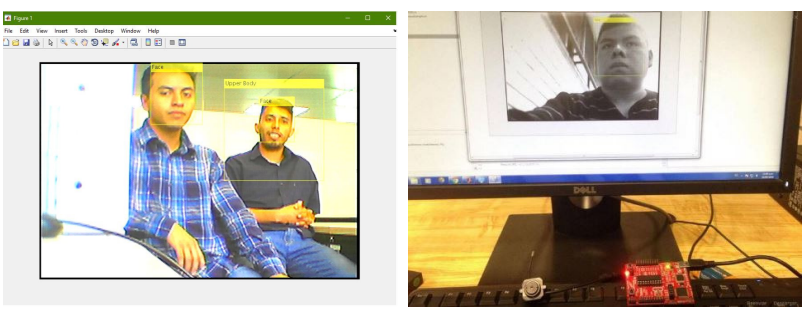

Fig. 7. Detección de rostro y parte superior del cuerpo, además de su representación en una la herramienta de desarrollo LaunchPad.

\section{Resultados}

1. Detección de rostro y parte superior del cuerpo, Figura 7.

2. Medir la distancia hacia la persona. Ya estando listo el sistema visión estereoscópico se colocó el sistema enfocando hacia una persona, después se ejecutó el programa en matlab y como se encontró persona. La distancia real medida desde las cámaras hacia la persona era $1 \mathrm{~m}$, la distancia calculada fue de $90 \mathrm{~cm}$.

3. Enlazar y desenlazar el transmisor con el receptor en el UAV para el control desde una computadora.

4. Aplicación de búsqueda de personas: Detectando el rostro de personas en un escenario de búsqueda y rescate, Figura 8.

Dentro de la aplicación introducimos el puerto serial a utilizar, en este caso usamos el puerto 3 y damos click en el botón OPEN. Ahora para enlazarnos con el UAV damos clic en el botón ON del panel ARMED.

Al dar clik en el botón START del panel SEARCHING el UAV comienza a elevarse verticalmente y posteriormente gira sobre su propio eje. Cuando el UAV detecta a una persona, esté avanza hacia ella si la distancia es menor de $2 \mathrm{~m}$ el UAV se detiene, por seguridad de la persona encontrada de lo contrario el UAV continúa avanzando. En la ventana principal de la aplicación se muestra el rostro de la persona detectada además de indicarlo de manera textual y gráfica con un indicador de color verde. 

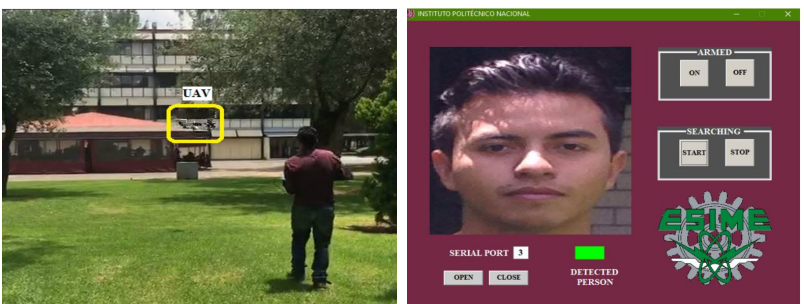

Fig. 8. Representación de la detección de un rostro.

\section{Conclusiones}

El presente trabajo propuso un sustema formado por dos subsistemas integrados, el primero un UAV controlado por un sistema computarizado, en tanto que el segundo es un sistema de visión por computadora que detecta la distancia cuando se encuentra una persona. El trabajo futuro de este sistema es la puesta en marcha para ser usado en un bosque, por ejemplo. Con ello se corregirán parametros que en laboratorio no se han tomado en cuenta.

Agradecimientos. Este trabajo es desarrollado con recursos e instalaciones del Instituto Politécnico Nacional, México por medio de los Proyectos SIP-20160786, SIP-20161053 y SIP-20161713. Además, fue apoyado por la Comisión de Operación y Fomento de Actividades Académicas del IPN (COFAA) y Consejo Nacional de Ciencia y Tecnología de México (CONACyT) por medio del Sistema Nacional de Investigadores con las becas No. 56739 (Dr. Moreno), 32772 (Dr. Morales) y 335839 (Dr. Tejeida). Cabe resaltar que este trabajo es parte de la tesis de Nivel Licenciatura de los alumnos Jesús Cruz, y Edgar Domínguez, dirigidos por el Dr. Jesús Jaime Moreno Escobar.

\section{Referencias}

1. Bertrand, S., Hamel, T., Piet-Lahanier, H.: Stabilization of a Small Unmanned Aerial Vehicle Model without Velocity Measurement. In: Proceedings 2007 IEEE International Conference on Robotics and Automation. pp. 724-729 (2007)

2. Lang, S.R., Luerssen, M.H., Powers, D.M.W.: Evolutionary Feature Preselection for Viola-Jones Classifier Training. In: Engineering and Technology (S-CET), 2012 Spring Congress on. pp. 1-4 (2012)

3. Lawlor, O., Moss, M., Kibler, S., Carson, C., Bond, S., Bogosyan, S.: Search-and rescue robots for integrated research and education in Cyber-Physical Systems. In: e-Learning in Industrial Electronics (ICELIE), 2013 7th IEEE International Conference on. pp. 92-97 (2013)

4. Paiva, E.A., Soto, J.C., Salinas, J.A., Ipanaqué, W.: Modeling and PID cascade control of a Quadcopter for trajectory tracking. In: 2015 CHILEAN Conference on Electrical, Electronics Engineering, Information and Communication Technologies (CHILECON). pp. 809-815 (2015) 
White-donkey: búsqueda de personas con vehículos aéreos no tripulados basada en visión ...

5. Zhang, Z.: A flexible new technique for camera calibration. IEEE Transactions on Pattern Analysis and Machine Intelligence 22(11), pp. 1330-1334 (2000) 